\title{
Fault Detection of an Internal Combustion Engine through Vibration Analysis by Wavelets Transform
}

\author{
Gina P. Novillo ${ }^{\mathrm{a}, 1}$, Néstor Diego Rivera Campoverde ${ }^{\mathrm{a}, 2}$, Héctor Adrian Auquilla Veintimilla ${ }^{\mathrm{a}, 3}$, \\ César Daniel Beltrán Orellana ${ }^{\mathrm{a}, 4}$ \\ ${ }^{a}$ GIIT, Universidad Politécnica Salesiana, Calle Vieja 12-30 y Elia Liut, Cuenca, 010105, Ecuador \\ E-mail: ${ }^{1}$ gnovillo@est.ups.edu.ec; ${ }^{2}$ nrivera@ups.edu.ec; ${ }^{3}$ aauquilla@est.ups.edu.ec; ${ }^{4}$ cbeltrano@est.ups.edu.ec
}

\begin{abstract}
This paper presents a vibration analysis of an internal alternative combustion engine through frequency analysis and wavelet transform, where a form study of the temporary signal and the energy of that signal is carried out to extract certain characteristic values that allow to differentiate and identify to which pre-established operating conditions, a specific vibration signal belongs. Software is used to make the data decomposition, analysis and value extraction. Different analysis results are presented on this investigation like frequency analysis, spectrogram analysis, wavelet analysis, cross wavelet analysis, and results validation by extracting values of the signals of two tests generating a variation chart showing runs variability if it is big o tiny variability. This analysis is performed to characterize the engine vibration signals so that it is possible to identify an incipient failure in a non-intrusive manner and optimize its maintenance. Also, it can be determined the repetitive form that describes a temporary signal of mechanical vibrations of a motor, if its work cycle it is considered to separate the temporary signal into sections, as long as there are no lower frequency components than the result of dividing the sampling frequency for the number of points that are in a work cycle (the limit frequency).
\end{abstract}

Keywords - fault detection; FFT; internal combustion engine; spectrogram; wavelets transform.

\section{INTRODUCTION}

Automotive industry research and industry in general is constantly carried out aiming improve systems performance [1]. Nowadays techniques and vibration analysis have been developed to optimize predictive maintenance of rotary machines, efficient and good results are noted especially in automatic detection of bearing defects.[2]-[9]. The application of Wavelet Transform (WT) for machine fault diagnostics has been developed for the last ten years at a very rapid rate [10]. Wavelet analysis is a mathematical technique used to represent data or functions [11]. Wavelet Transform has gained acceptance in the signal processing being a processing technique that could be used to represent non-stationary signals of real life with high efficiency [12]; it has demonstrate its use in rotary machines fault diagnosis [13] and it can be categorized as continuous wavelet transform (CWT); discrete wavelet transform (DWT), and wavelet packet transform (WPT), it should be taken into account that in all these methods an inappropriate wavelet basis used in an application will directly influence the accuracy of the fault diagnosis. [14]

In [15] is showed the possibility of diagnosing damage to the timing chain tensioner in a motorcycle engine, applying vibration signal processing by means of a CWT; DWT has been applied in [16]; on vibrational signals acquired by an accelerometer for detecting knock phenomena, the same analysis using Maximum Amplitude Pressure Oscillation (MAPO) has been done in [17] that is applied to pressure data, both investigations are compared and it confirms that DWT use represents a very valid alternative to the traditional knock detection techniques; [18] presents a combined technique of ensemble empirical mode decomposition (EEMD), robust independent component analysis (RobustICA) and continuous wavelet transform (CWT) to identify the origin of gasoline engine noise.

Fault diagnosis system for automotive generators using DWT and an artificial neural network is described in [19] which results indicate that mentioned diagnosis is effective while in [20] is presented a fault diagnosis system for internal combustion engines using wavelet packet transform (WPT) and artificial neural network (ANN) techniques; and a fault signal diagnosis technique for internal combustion engines that uses a continuous wavelet transform algorithm is carried out in [21].

Most research is aimed at testing hypotheses or data already known as cylinder pressure, noise or emissions, therefore, the need and importance of implementing these methods to determine the operating state of an alternative 
engine based on the acquisition and analysis of certain operating data, for which it has been considered develop a technical method that helps to discretize the signals that emit these failures.

\section{MATERIAL AND METHOD}

\section{A. Experimental Design}

Table I shows the used methodology which is done in one of the signals of an experimental design that consist of 5 variables at 3 levels each one which can be manipulated during the essay at the moment of the data acquisition in that way put the effect in the response variable that is required to analyze [22]

TABLE I

FACTORS AND LEVELS OF THE EXPERIMENTAL DESIGN

\begin{tabular}{|l|l|l|l|l|}
\hline \multirow{2}{*}{ Variables } & \multirow{2}{*}{ Unit } & \multicolumn{3}{|c|}{ Levels } \\
\cline { 3 - 5 } & & Minimum & Medium & Maximum \\
\hline Fuel Pressure & $\mathrm{Mpa}$ & 0.125 & 0.3 & 0.475 \\
\hline Air Filter Capacity & $\%$ & 25 & 62.5 & 100 \\
\hline $\begin{array}{l}\text { Catalyst } \\
\text { Obstruction }\end{array}$ & $\mathrm{cm}^{2}$ & 7.54 & 14.8 & 22.06 \\
\hline $\begin{array}{l}\text { Spark plugs badly } \\
\text { calibrated }\end{array}$ & $\mathrm{Mm}$ & 0.8 & 1 & 1.2 \\
\hline Rate of rotation & $\mathrm{rpm}$ & 760 & 1630 & 2500 \\
\hline
\end{tabular}

\section{B. Data Acquisition}

1) Semi-anechoic Chamber: To obtain data of a reliable way and without strange noises intrusion or disturbances [23], tests were performed in the Universidad Politécnica Salesiana semianechoic chamber.

2) Monitoring Equipment: As a data monitoring equipment for the realized tests, ADASH A3716 was used. This apparatus has $16 \mathrm{AC}$ channels and 16 DC channels which they can work together to obtain several signals at the same time managing to visualize in real time and in turn save in the system. With an option of the equipment, registered signals can be transformed into audio format archive.wav of various channels for later analysis and manipulate by means of Matlab.

3) Accelerometer: For the correct placement of the accelerometer was used: ISO 10816-6:1995 mechanical vibration - evaluation of machine vibration by measurements on non-rotating parts - part 6: Reciprocating machines with power ratings above $100 \mathrm{~kW}$ which establishes placing it on the side, perpendicular to the longitudinal axis of the engine [24]. The accelerometer that was used is A102-1A model, its characteristics is shown in Table II.

TABLE II

FACTORS AND LEVELS OF THE EXPERIMENTAL DESIGN

\begin{tabular}{|l|l|}
\hline Sensor & $\mathrm{A} 102-1 \mathrm{~A}$ \\
\hline Nominal sensitivity & $100 \mathrm{mV} / \mathrm{g}$ \\
\hline Calibration method & $\mathrm{ISO} 16063-21: 2003$ \\
\hline Calibration result & $\mathrm{K}=82 \mathrm{~Hz}$ \\
\hline Constant $[\mathbf{k}]+/-3 \%$ & 1,02 \\
\hline Sensitivity $[\mathbf{m V} / \mathbf{g}+/-3 \%]$ & 102 \\
\hline BIAS $[\mathbf{V}]+/-2 \%$ & 11,9 \\
\hline
\end{tabular}

4) Inductive Clamp: Jointly to check obtained data, it was necessary to acquire data from the spark jump of the first cylinder for which an inductive clamp was placed to the spark plug wire of the cylinder.

\section{Data Nomenclature}

In Fig. 1 the coding given to each sample can be observed based on the different parameters, in order to generate a database that is more accessible.

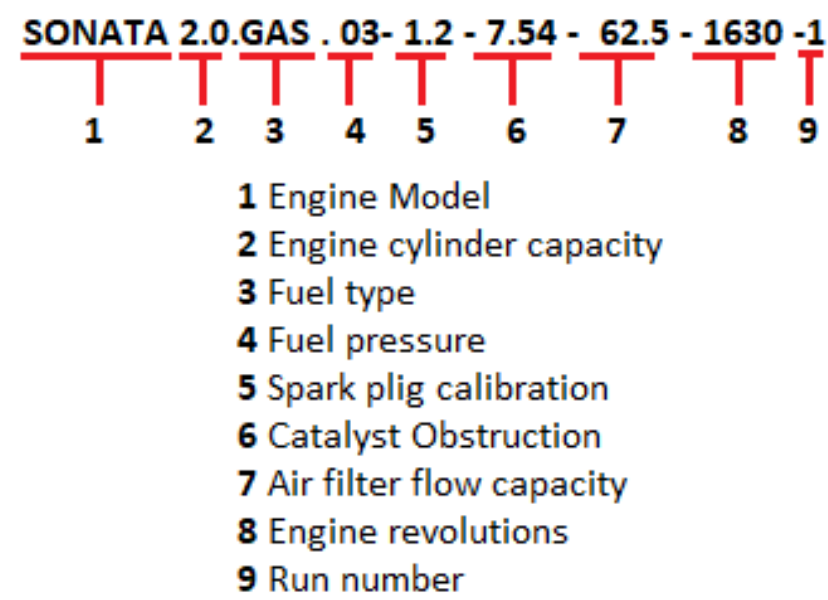

Fig. 1 Signals Nomenclature

Table I details parameters of each one of the factors, in such a way can be observed minimum, intermediate and maximum values.

\section{Analysis Method}

The main objective of analyze obtained results of the tests, is to extract from them the greatest quantity of characteristic numerical values, allowing them to be differentiated from each other and therefore establish necessary characteristics of a signal that corresponds to a certain pre-establish engine failure.

For this, is established to use Fast Fourier Transform (FFT) which helps to determine the fundamental frequency of a $\mathrm{Hz}$ signal. Applying spectrogram analysis and Wavelet transform to the signals, it can be observed in Fig. 2 a signal of the tests "SONATA2.0.GAS.0,3-0,8-14,8-62,5-760".

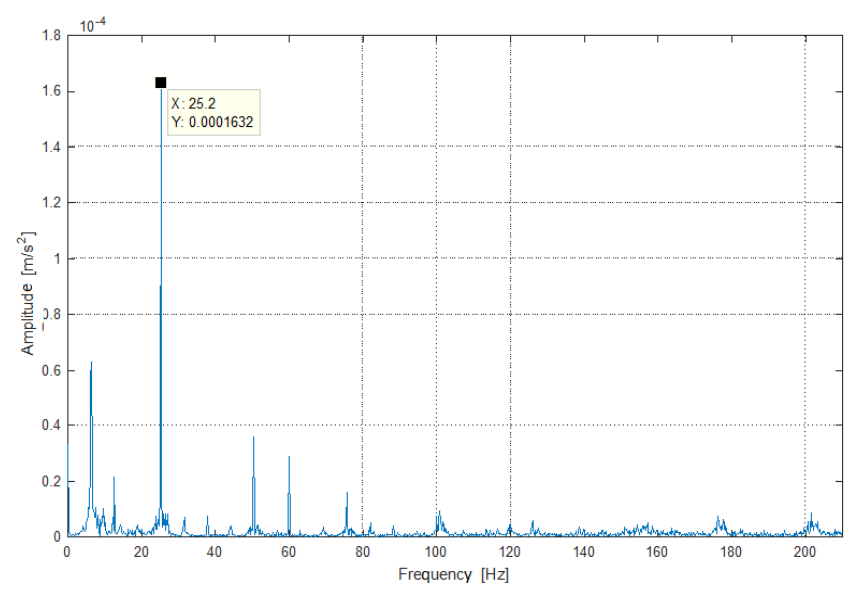

Fig. 2 FFT of a signal at $760 \mathrm{rpm}$ 
Figure 2 shows the fundamental frequency of the signal being of $25.2 \mathrm{~Hz}$, also it can be observed different irregularities at the base of the peaks which may have important information or maybe external disturbances, for this purpose it was considered to perform an optimization of the frequency spectrum of the FFT.

1) Frequency Spectrum Optimization: To optimize the frequencies analysis and the peak detection a signal homogenization is performed which consist in matching the duration of each work cycle (2 crankshaft turns) to a constant length. In Fig. 3 is observed the extracted accelerometer signal, with a sampling frequency of $\mathrm{Fs}=65536$.

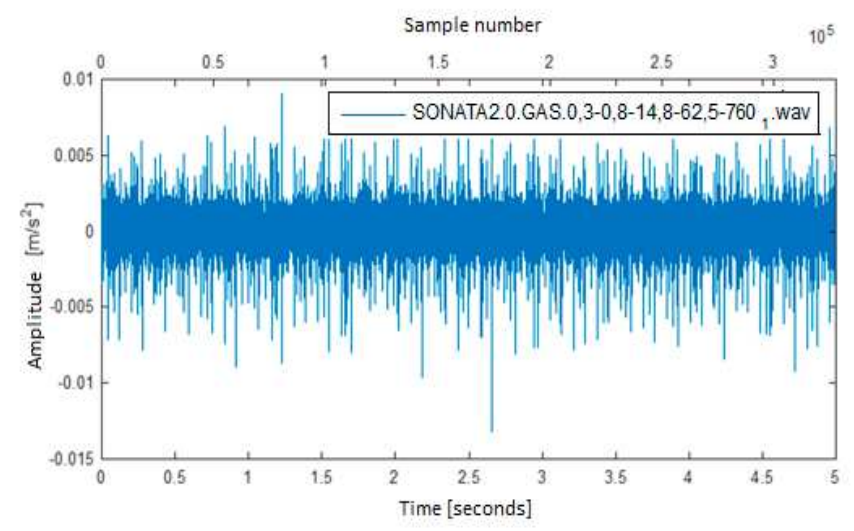

Fig. 3 Accelerometer temporary signal

In order to determine the exact moment in which begins and ends a work cycle, reference is made at the instant in which the spark jumps to the cylinder number one, for this, the inductive clamp is used which allows to obtain the temporary signal of the voltage induced by the spark plug wire of the cylinder number one as seen in Fig. 4.

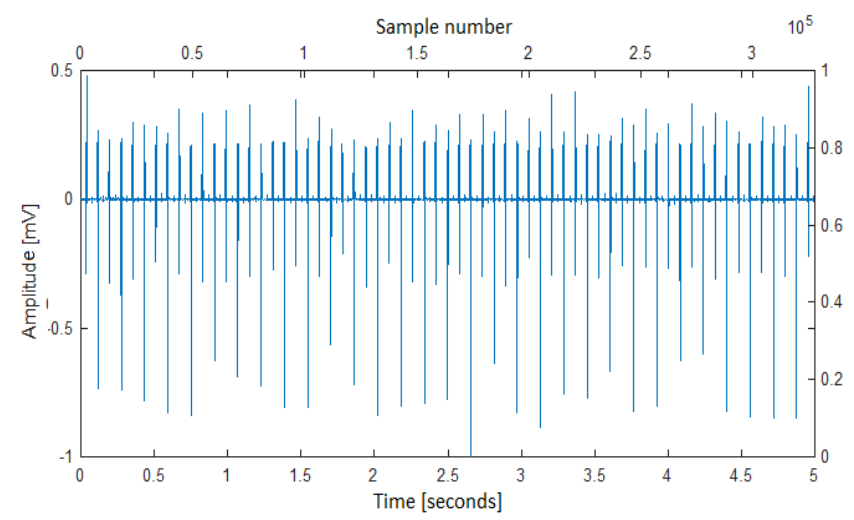

Fig. 4 Inductive Clamp temporary signal

To determine the moment at which each work cycle begins and ends, the fact that it is being treated with a semisequential ignition system is considered, therefore the highest peaks determine the jump of the spark. To detect the peaks of this signal, only the absolute value of the processed signal is used, and the Symlet4 wavelet is applied, since it has a symmetry that allows easy peaks detection and a very good coupling at the peak of the signal.
Fig. 5 shows that it has been possible to determine maximum points (red dots) in the instant that the work cycle is fulfilled, that is, when the spark jump occurs, while lower points indicate the jump of the spark in cylinder 4 (blue dots).

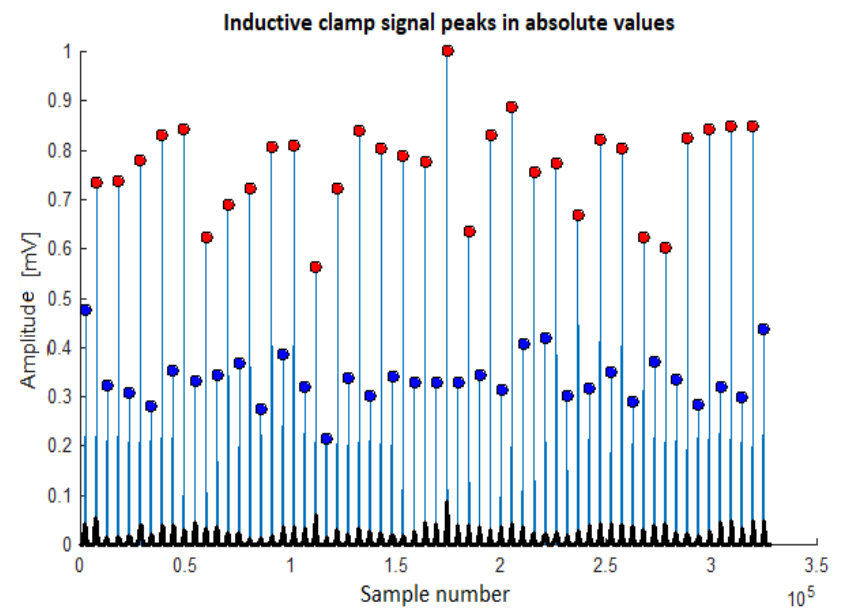

Fig. 5 Signal peaks of the inductive clamp

Once the indexes of the inductive clamp signal have been extracted, they are checked with the accelerometer signal, thereby dividing the signal into work cycles as indicated in Fig. 6a, managing to visualize that mentioned signals tend to maintain the same form, but because the rpm is not constant the signals tend to have different lengths, however all the signals can be adjusted to the same length by adding zeros as an amplitude value in a distributed manner without altering the shape of the signal, obtaining in such a way the signals of the work cycles with the same lengths as seen in Fig. 6 b.

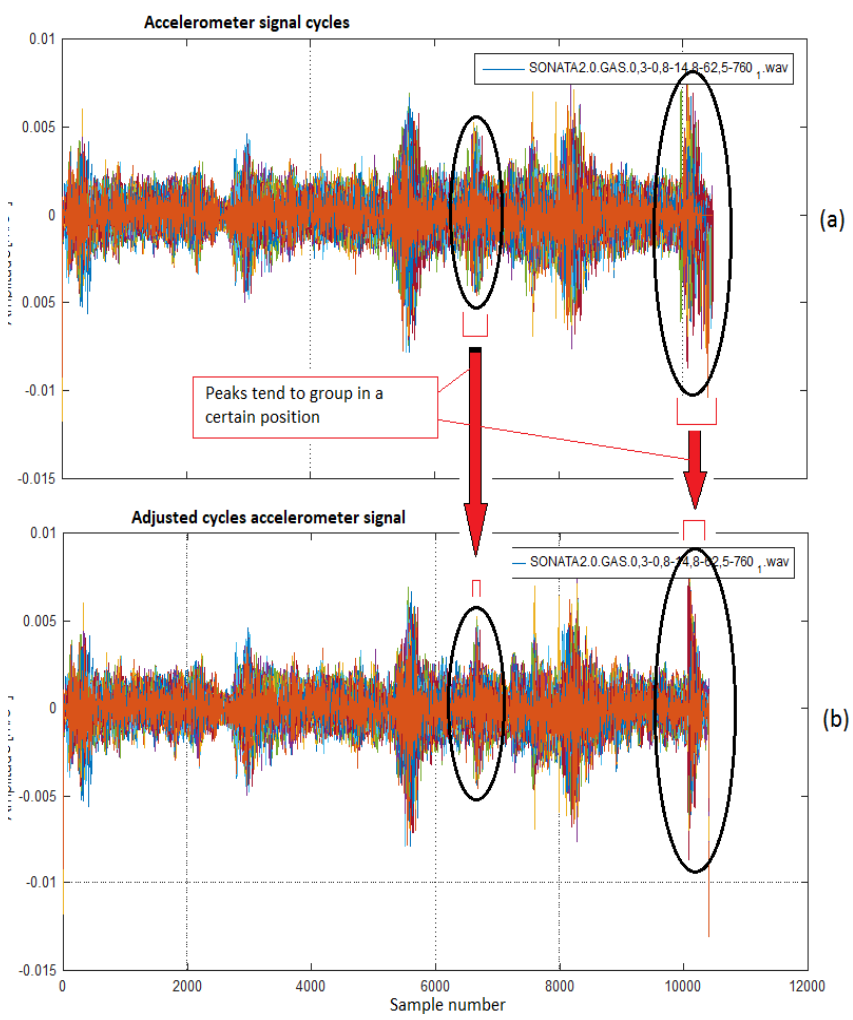

Fig. 6 Signal divided into work cycles 
When matching sections of the signal corresponding to the engine work cycles, to the same length, it is noted that the signal peaks tend to be grouped describing clearly the shape of the repetitive pattern of the signal; applying FFT it could be observed in Fig. 7 that the optimized signal has fewer irregularities compared to the original signal but maintaining the frequency peak.

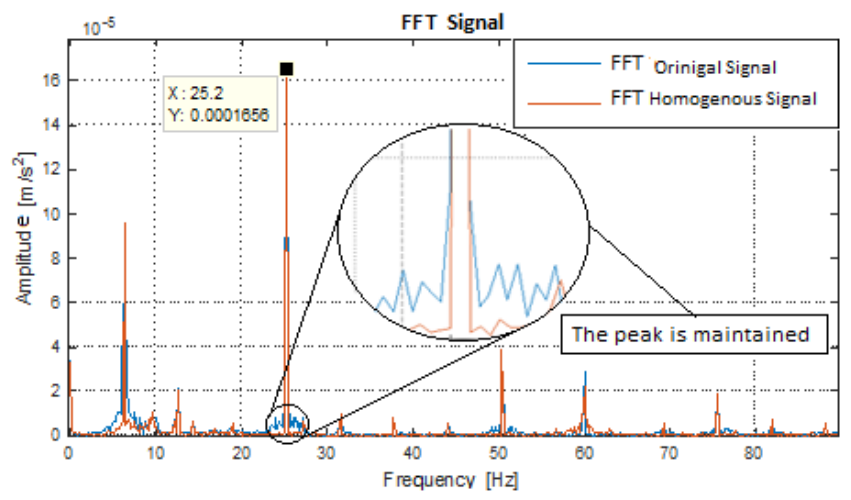

Fig. 7 Signal Homogenization in FFT

In addition, depending on the engine speed, the signals reduce irregularities as observed in Fig. 8 where the optimization process in the FFT has been applied in signals with different rpm.
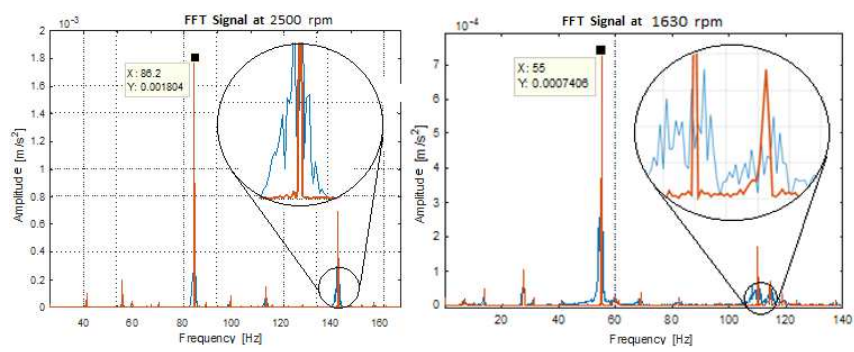

Fig. 8 Optimization effect on the FFT at 2500 and $1600 \mathrm{rpm}$

The main characteristic values of the frequencies and amplitude that make up the signal are thus obtained; criteria for the detection of these values or peaks are: permissible amplitude, peak band, Wavelet family, Decomposition level and frequency range

Obtained characteristic values are shown in Table III, Subsection III-A

2) Analysis with Spectrogram: The signal in the frequency domain is decomposed but without losing the time axis, thus be able to observe the changes in the frequency of the signal as a function of time, for this the FFT is used in very small intervals and it is formed a mantle of frequencies as a function of time. The frequency for a specific number of points is represented by Equation 1

$$
\text { finit }=\frac{F s}{\text { number of points }}
$$

Therefore, $f_{\text {inmit }}=6.3 \mathrm{~Hz}$. This $6.3 \mathrm{~Hz}$ means that values with a high frequency than this one is within the 2 crankshaft turns that corresponds to an engine work cycle; while that frequencies under $6.3 \mathrm{~Hz}$ correspond to events that occur more than two crankshaft turns. Fig. 9 shows a spectrogram sample of the signal SONATA2.0.GAS.0,3-0,8-14,8-62,5760 , where is identified the fundamental frequency of the signal (red line), limit frequency (black line), and the main frequencies that are maintained with a small variation range during all the test, these frequencies are the same that were determined with the FFT.

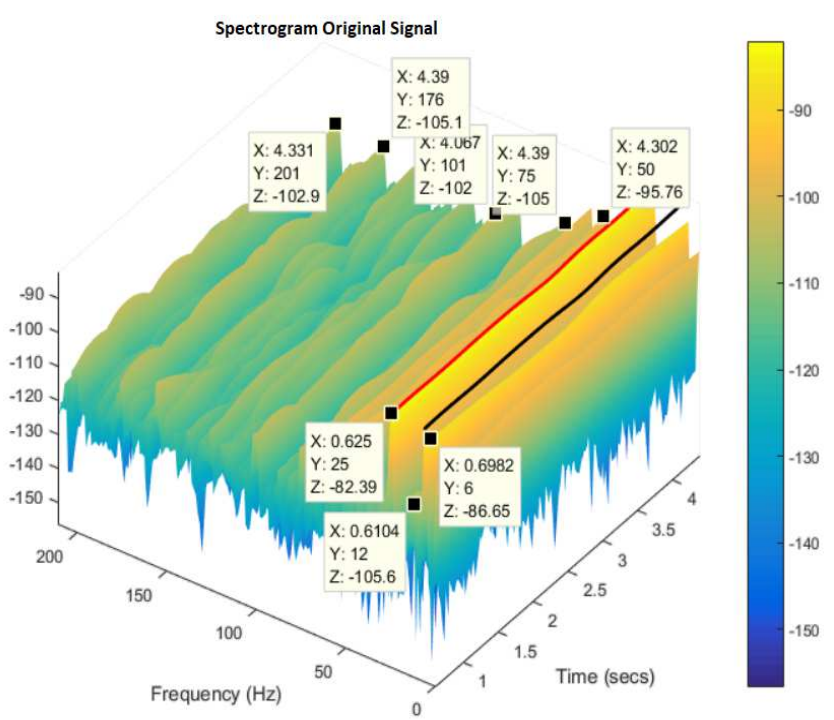

Fig. 9 Spectrogram Signal

The analysis result with spectrogram is detailed in subsection III-B

3) Wavelet Transform: In Fig. 10 is presented a vibration signal for the SONATA2.0.GAS.0,3-0,8-14,8-62,5-760 test, which is divided into work cycles and as a function of crankshaft turn, during the test a $25^{\circ}$ advance in the ignition was registered by the OBDII port, which also determines the crankshaft PMS and the ignition timing for each engine cylinder.

Signal divided into engine work cycles

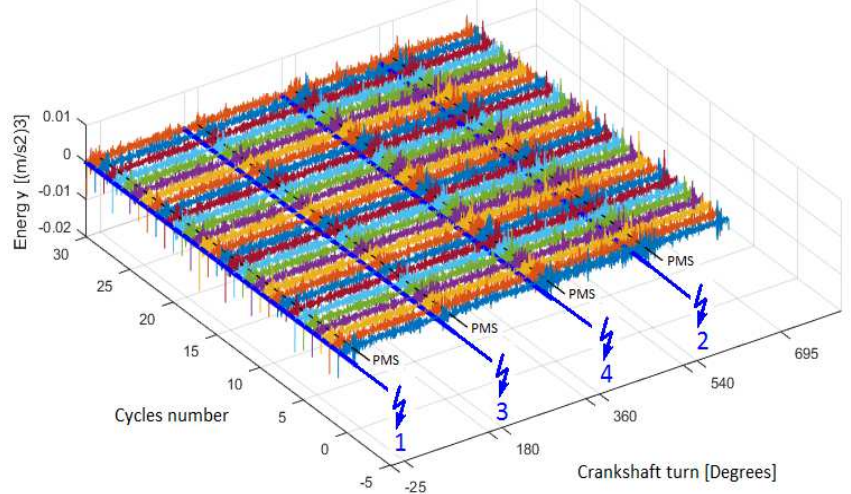

Fig. 10 Signal divided into work cycles

For determining the representative shape of the signal, the optimization method described above is applied, in which the length of the work cycles is equalized in order to group the signal peaks and better define their shape, a weighted average is used to determine the representative shape of the signal (Fig. 11) 


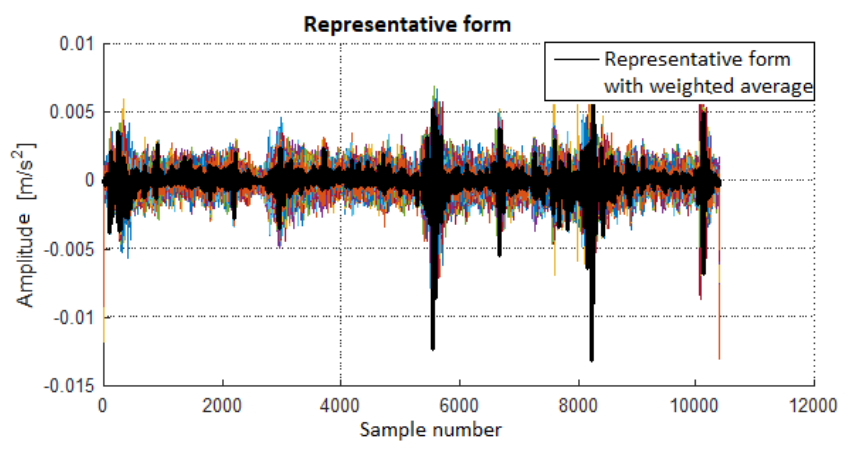

Fig. 11 A representative form of the signal

Once obtained the representative form of the signal, the continuous Wavelet transform (CWT) is carried out as observed in Fig. 12.

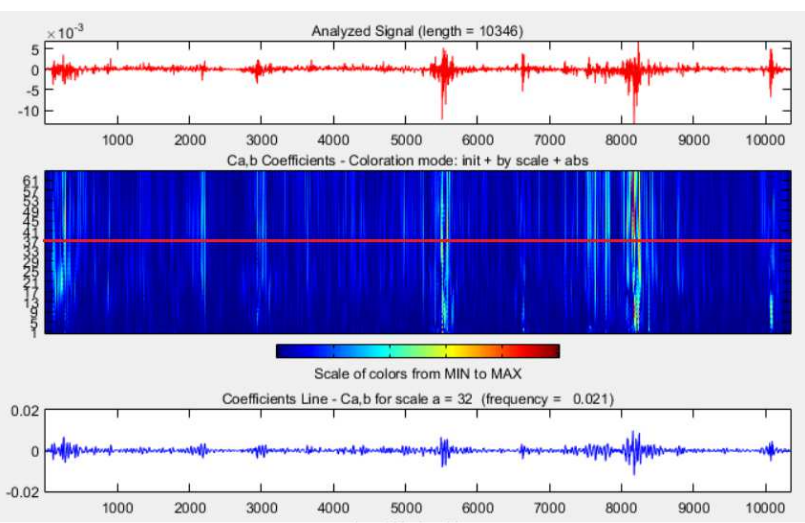

Fig. 12 CWT of the representative shape of the signal

Results are detailed in subsection III-C

4) Analysis of the energy of the signal: Estimated spectral density of the signal is determined in order to localize the frequency component with greater power, since the highest amount of the energy of the signal is found in this frequency; Fig. 13 shows the distribution of the signal power in function of the frequency.

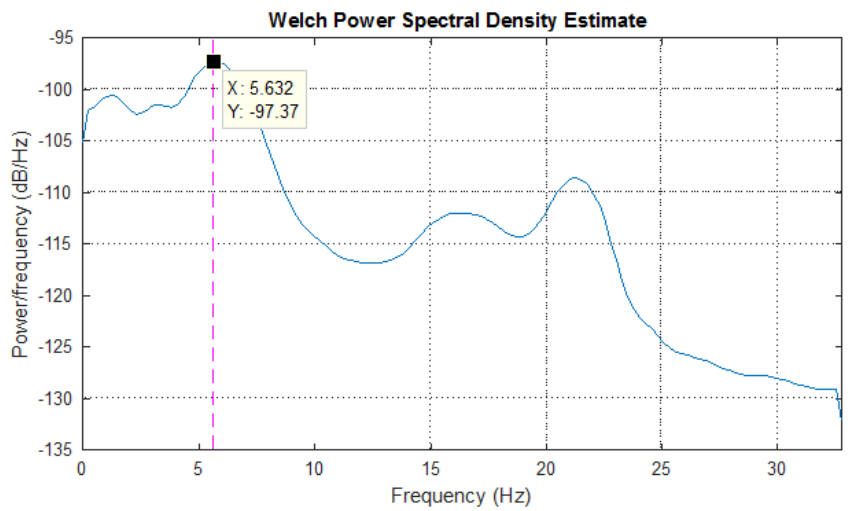

Fig. 13 Estimated Density of signal power

The maximum value $5.632 \mathrm{~Hz}$ corresponds to the frequency of the highest energy content of the signal, with this value is determined where the higher spectral information is found, to find the signal points with greatest energy content inside this frequency, the CWT is used from which a contour plot is obtained showing the level curves that indicate the energy of the signal.

To determine the scale of expansion coefficient of the CWT where is located the maximum energy points, in this case the Daubechies (db2) second derivative is used since it presents asymmetry and little regularity in its form which it allows a good couple to the original shape of the accelerometer signal. For the $\mathrm{db} 2$ wavelet, a relationship curve is drawn between its fundamental frequency and its changes depending on the scale or coefficient of expansion, so that with the frequency of higher energy content of the studied signal, that is $5.632 \mathrm{~Hz}$, the corresponding scale of the wavelet used where the highest energy content of the signal will be found is also determined, as indicated in Fig. 14.

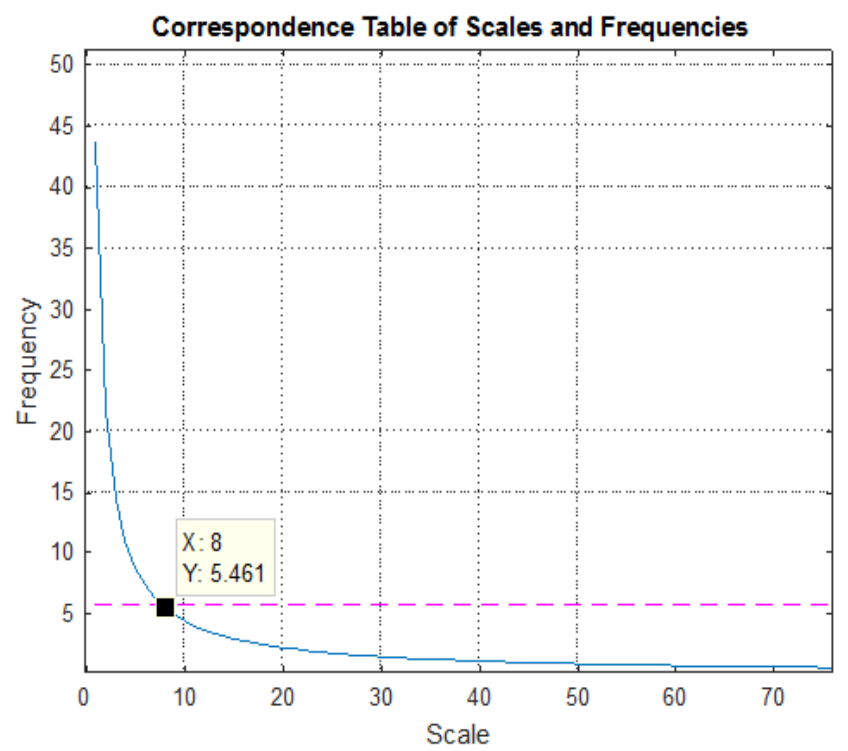

Fig. 14 Scale correspondence for Wavelet $\mathrm{db} 2$ at the set frequency

The highest energy and information content will be located around scale 8, Fig. 15 shows the CWT of the signal and the scale with the highest energy content.
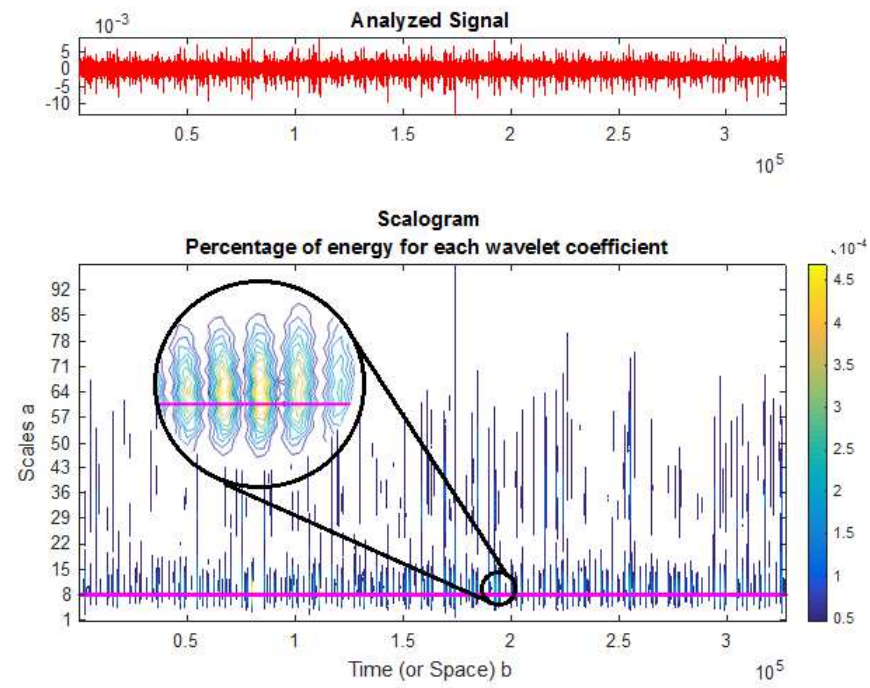

Fig. 15 CWT test accelerometer signal 
5) Cross Wavelet Analysis and signal phase coherence: Analysis by means of crossed CWT between two temporal signals allows us to determine the similarities between the mentioned signals by the local covariance factor of the scaletime spectrum. For this, complex wavelets are used, as this can also determine the offset between the signals. The characteristic shape of a signal is taken then is applied the Wavelet crossed transform and it is determined at which scale of the scale-time plane the highest covariance factor is found and in which scale the lowest covariance factor. Fig. 16 shows the scale-time plane of the crossed Wavelet transforms for the signal of two runs belonging to the same test.
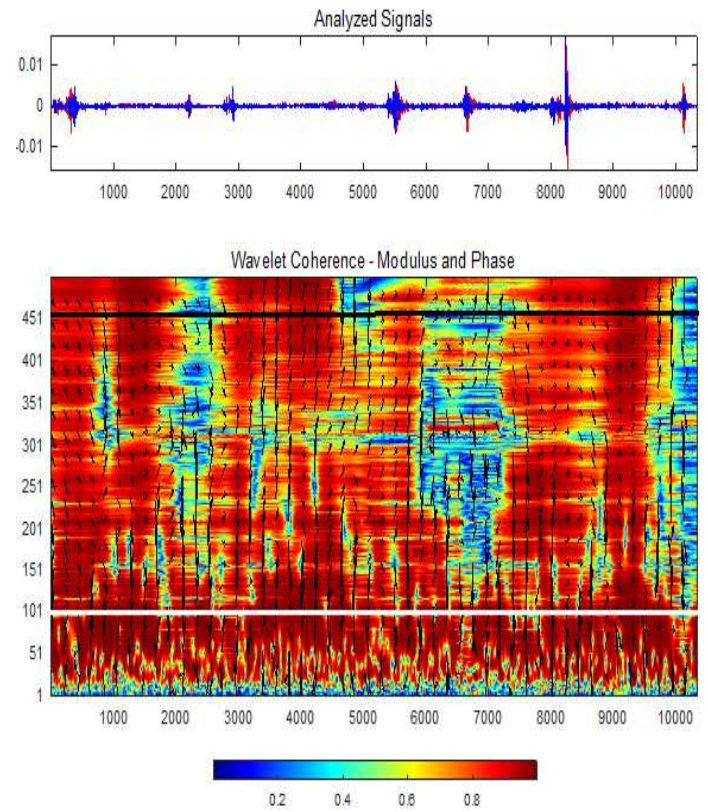

Fig. 16 Cross Wavelet and coherence between signals of the same test

In Fig. 17 is observed the scale-time plane of the crossed Wavelet transform for the signal of two runs belonging to different tests SONATA2.0.GAS.0,3-1-22,06-100760_1_STD and SONATA2.0.GAS.0,125-1-14,8-62,5760_1. In the same way, the red zones indicate the similarity between signals and blue zones the difference between them.
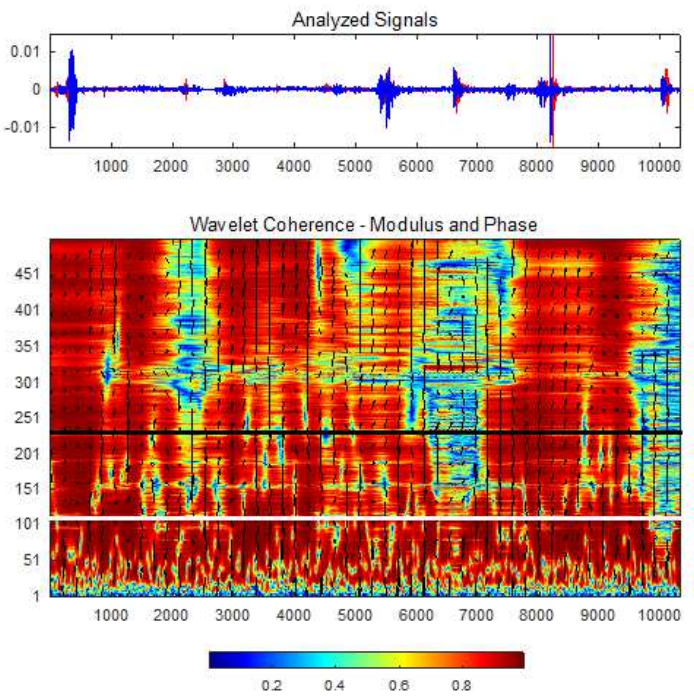

Fig. 17 Cross Wavelet and coherence between signals of different tests.
Results of this process are shown in subsection III-D

\section{RESULTS AND DISCUSSION}

\section{A. Frequency Analysis Results}

Obtained characteristic values from the frequency analysis of the signal SONATA 2.0.GAS.0,3-0,8-14,8-62,5760 is indicated in Table III.

TABLE III

FREQUENCY CHARACTERISTIC VALUES

\begin{tabular}{|l|l|l|l|l|l|}
\hline \multicolumn{2}{|c|}{ Values for detection } & \multicolumn{3}{c|}{ Harmonics } \\
\hline Criterion & Value & Unit & Type & Frequency & Amplitude \\
\hline $\begin{array}{l}\text { Minimum } \\
\text { permissible } \\
\text { amplitude } \\
\text { based on } \\
\text { maximum } \\
\text { amplitude }\end{array}$ & 10 & $\%$ & $\begin{array}{l}\text { Ff } \\
0,25 \mathrm{X}\end{array}$ & $\begin{array}{l}25.2 \\
6.3\end{array}$ & $\begin{array}{l}0.0001656 \\
9,55 \mathrm{E}-02\end{array}$ \\
\hline $\begin{array}{l}\text { Medium } \\
\text { band for } \\
\text { local } \\
\text { maximum } \\
\text { detection }\end{array}$ & 1 & $\mathrm{~Hz}$ & $2 \mathrm{X}$ & 50.4 & $3,89 \mathrm{E}-02$ \\
\hline $\begin{array}{l}\text { Frequency } \\
\text { Domain }\end{array}$ & $0-210$ & $\mathrm{~Hz}$ & $7 \mathrm{X}$ & 176.4 & $1.88 \mathrm{e}-5$ \\
\hline
\end{tabular}

\section{B. Spectrogram Analysis Results}

Spectrogram analysis could bear out with the existence of approximately stationary frequencies during the test, which provides information and allows to verify Fourier analysis reliability since in this the same frequencies determined with the FFT are found and are maintain along the test time.

\section{Wavelet Analysis Results with Respect to the Signal Shape}

The result of this procedure is to obtain a cleaner signal and that retains most of the information of the original signal, from this signal can be extracted main characteristics of a signal as shows in table IV.

TABLE IV

ChaRACTERISTIC VALUES RESPECT TO THE SignAL

\begin{tabular}{|c|c|c|c|}
\hline \multicolumn{3}{|c|}{ Values for signal detection } & \multirow{2}{*}{$\begin{array}{l}\text { Characteristics } \\
\text { Kurtosis }\end{array}$} \\
\hline Type & Value & $\begin{array}{l}\text { Crankshaft } \\
\text { Turn }\end{array}$ & \\
\hline \multirow[t]{4}{*}{ Wavelet used } & \multirow{4}{*}{$\begin{array}{l}\text { Daubechies } \\
\text { Second } \\
\text { derivative } \\
(\mathrm{db} 2)\end{array}$} & $90^{\circ} \mathrm{x} 1$ & 23,5433702 \\
\hline & & $90^{\circ} \mathrm{x} 2$ & 25,3617317 \\
\hline & & $90^{\circ} \mathrm{x} 3$ & 25,4486649 \\
\hline & & $90^{\circ} \mathrm{x} 4$ & 6,62694988 \\
\hline \multirow{4}{*}{$\begin{array}{l}\text { Predominant } \\
\text { scale }\end{array}$} & \multirow[t]{4}{*}{32} & $90^{\circ} \times 5$ & 22,0677896 \\
\hline & & $90^{\circ} \times 6$ & 40,0476393 \\
\hline & & $90^{\circ} \times 7$ & 68,841636 \\
\hline & & $90^{\circ} \mathrm{x} 8$ & 43,2772008 \\
\hline
\end{tabular}

D. Cross Wavelet Analysis Results and Coherence between Signals Phase

The program detects scales automatically and offset in which the two temporal signals are more coincident (white line (Fig. 16 and Fig. 17). The results of crossing equal signals and differences are shown in Table V and Table VI. 
TABLE V

CWT CROSS RESULTS FOR THE SIGNALS SONATA2.0.GAS.0,3-1-22,06100-760_1_STD AND SONATA2.0.GAS.0,3-1-22,06-100-760_2 STD

\begin{tabular}{|l|l|l|l|l|}
\hline \multirow{2}{*}{ Cross Signals } & \multicolumn{2}{|l|}{ Maximum Coherence } & \multicolumn{2}{l|}{ Minimum Coherence } \\
\cline { 2 - 5 } & Covariance & Offset & Covariance & Offset \\
& & & & \\
\hline $\begin{array}{l}\text { SONATA2.0.GAS.0,3- } \\
\text { 1-22,06-100- } \\
\text { 760_1_STD }\end{array}$ & & & & \\
\cline { 1 - 2 } & 0,984 & 0,482 & 0,621 & 0,292 \\
1-22,06-100- & & & & \\
760_2_STD & & & & \\
\hline
\end{tabular}

TABLE VI

CROSS RESULTS FOR THE SIGNALS SONATA2.0.GAS.0,3-1-22,06-100760_1_STD AND SONATA2.0.GAS.0,125-1-14,8-62,5-760_1

\begin{tabular}{|l|c|c|c|c|}
\hline \multirow{2}{*}{ Cross Signals } & \multicolumn{2}{|c|}{$\begin{array}{c}\text { Maximum } \\
\text { Coherence }\end{array}$} & \multicolumn{2}{c|}{$\begin{array}{c}\text { Minimum } \\
\text { Coherence }\end{array}$} \\
\cline { 2 - 5 } & Covariance & Offset & Covariance & Offset \\
\hline $\begin{array}{l}\text { SONATA2.0.GAS.0,3-1- } \\
\text { 22,06-100-760_1_STD }\end{array}$ & \multirow{2}{*}{0,979} & 0,058 & 0,700 & 0,403 \\
\cline { 1 - 1 } $\begin{array}{l}\text { SONATA2.0.GAS.0,125- } \\
\text { 1-14,8-62,5-760_1 }\end{array}$ & & & & \\
\hline
\end{tabular}

\section{E. Results Validation:}

For the validation of the results, extracted values of the signals of two tests and its respective runs are taken, generating a variation chart showing that the values between runs have little variability, while between different tests there is greater variability. Variability between tests is very small, this is because it is being treated with incipient failures whose effects are tiny but demonstrable.

Fig. 18 shows the cash graph of the obtained characteristic values with the FFT for each one of the runs of the tests SONATA2.0.GAS.0,3-1-22,06-100-760_STD y SONATA2.0.GAS.0,125-1-14,8-62,5-760_FAILURE,

although 5 of 9 parameters are separated, none has a considerable separation to be able to infer that there is some difference between the signals, consequently a signal belonging to the incipient faults established in the motor cannot be detected or characterized by the Fast Fourier Transform.

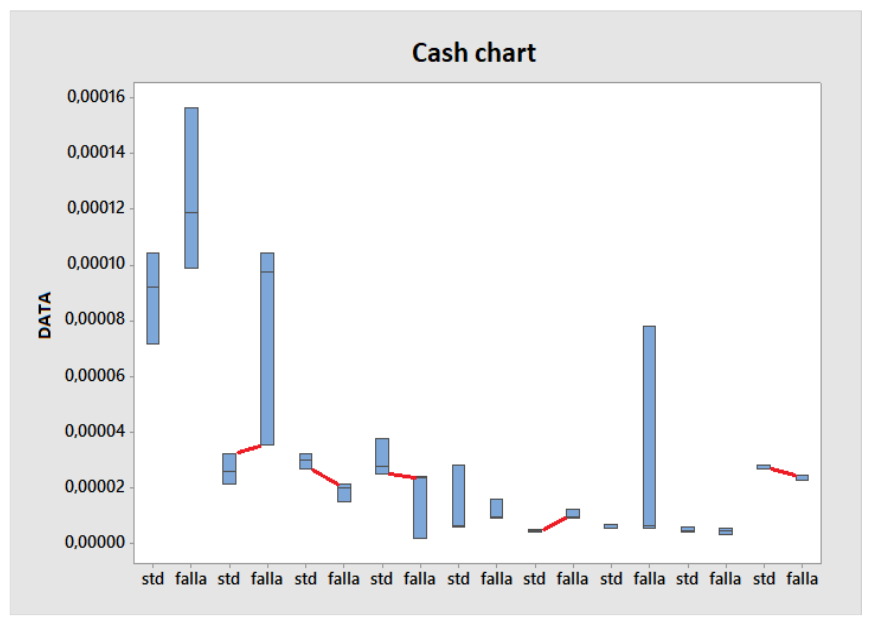

Fig. 18 Cash graph of the characteristic values by means of the FFT
In the case of the analysis shape of the signal by means of the Wavelet decomposition, Fig. 19 shows that 5 of the 16 parameters are separated and only one of them has considerable separation, for this reason the fact that this analysis does not exceed, in this case, the FFT method.

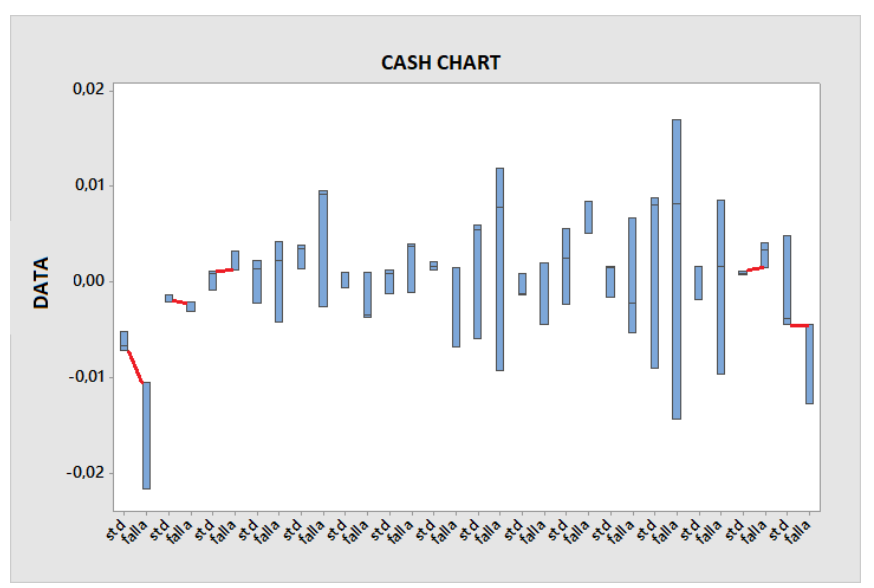

Fig. 19 Cash graph of the characteristic values by means of the FFT

\section{CONCLUSIONS}

The frequency spectrum analysis by means of fast Fourier FFT does not allow a signal to be significantly differentiated from another, so it does not constitute an adequate method for detection and characterization of tiny or incipient failures that are reflected in these vibration signals. The continuous CWT wavelet transform allows obtaining a plane time scale that magnifies the shape of a signal allowing a better way of observation of the influence of the signal undulations and determine the scale with greater energy content, therefore with more signal information.

It can be determined the repetitive form that describes a temporary signal of mechanical vibrations of a motor, if its work cycle it is considered to separate the temporary signal into sections, as long as there are no lower frequency components than the result of dividing the sampling frequency for the number of points that are in a work cycle (the limit frequency). The continuous wavelet transform allows to simplify the signal to its most compact expression and to keep most of its original characteristics, however it cannot be detected if the information lost is relevant or not.

When determining the repetitive shape of the vibration signal based on the rotation of the crankshaft and applying the wavelet transform to simplify the signal keeping most of its information, it was not possible to determine the characteristics that allow identifying an incipient failure.

The cross-wavelet transform allows to determining how much and at what time two signals are coherent or similar, as well as how and at what time the same signals are different. An experimental method that takes advantages of these qualities can be developed. The values extracted from the signals are not varied enough to generate a surface response but allowed to verify that they tend to differentiate so that this investigation can be continued using other methods. The incipient failures that were raised caused tiny effects on the mechanical vibrations of the motor, so they are almost undetectable but are the database for new research projects. 


\section{ACKNOWLEDGMENT}

The authors express gratitude to Engineer Nestor Rivera for all his unconditional support and contributing his knowledge to the realization of this paper. Also, the authors are thankful to the investigation group "Grupo de Investigación en Ingenieria de Transporte", GIIT, of the Universidad Politécnica Salesiana for all support during this investigation.

\section{REFERENCES}

[1] Néstor Rivera, Juan Chica, Ivan Zambrano, and Cristian Garc'1a. Estudio del comportamiento de un motor ciclo otto de inyeccion electronica respecto de la estequiometr'́1a de la mezcla y del adelanto al encendido para la ciudad de cuenca. Revista Polit'ecnica, 40(1):59-67, 2017.

[2] L Hinke, L Pichler, HJ Pradlwarter, BR Mace, and TP Waters. Modelling of spa- tial variations in vibration analysis with application to an automotive windshield. Finite Elements in Analysis and Design, 47(1):55-62, 2011.

[3] Yoshio Kurosawa, Hideki Enomoto, Shuji Matsumura, and Takao Yamaguchi. High frequency vibration analysis of automotive bodies with panels that have attached viscoelastic layers. In ASME 2003 International Mechanical Engineering Congress and Exposition, pages 23-29. American Society of Mechanical Engineers Digital Collection, 2003.

[4] Seyed Hamed Mirafzal, Amir Mahyar Khorasani, and Amir Hossein Ghasemi. Op- timizing time delay feedback for active vibration control of a cantilever beam using a genetic algorithm. Journal of Vibration and Control, 22(19):4047-4061, 2016.

[5] $\mathrm{T}$ Yamaguchi, Y Kurosawa, and $\mathrm{H}$ Enomoto. Damped vibration analysis using finite element method with approximated modal damping for automotive double walls with a porous material. Journal of Sound and Vibration, 325(1-2):436-450, 2009.

[6] Sze-jung Wu, Nagi Gebraeel, Mark A Lawley, and Yuehwern Yih. A neural net- work integrated decision support system for condition-based optimal predictive maintenance policy. IEEE Transactions on Systems, Man, and Cybernetics-Part A: Systems and Humans, 37(2):226-236, 2007.

[7] Zhiqiang Huo, Yu Zhang, Pierre Francq, Lei Shu, and Jianfeng Huang. Incipient fault diagnosis of roller bearing using optimized wavelet transform based multi- speed vibration signatures. IEEE Access, 5:19442-19456, 2017.

[8] Xinsheng Lou and Kenneth A Loparo. Bearing fault diagnosis based on wavelet transform and fuzzy inference. Mechanical systems and signal processing, 18(5):1077-1095, 2004.

[9] Stefan Ericsson, Niklas Grip, Elin Johansson, Lars-Erik Persson, Ronny Sjoberg, and Jan-Olov Stromberg. Towards automatic detection of local bearing defects in rotating machines. Mechanical systems and signal processing, 19(3):509-535, 2005.
[10] ZK Peng and FL Chu. Application of the wavelet transform in machine condition monitoring and fault diagnostics: a review with bibliography. Mechanical systems and signal processing, 18(2):199-221, 2004.

[11] Daljeet Kaur Khanduja and MY Gokhale. Time domain signal analysis using modified haar and modified daubechies wavelet transform. Signal Processing-An International Journal (SPIJ), 4(3):161, 2010.

[12] Dipalee Gupta and Siddhartha Choubey. Discrete wavelet transform for image processing. International Journal of Emerging Technology and Advanced Engineering, 4(3):598-602, 2015.

[13] Shuilong He, Yikun Liu, Jinglong Chen, and Yanyang Zi. Wavelet transform basedon inner product for fault diagnosis of rotating machinery. In Structural Health Monitoring, pages 65-91. Springer, 2017.

[14] Jinglong Chen, Zipeng Li, Jun Pan, Gaige Chen, Yanyang Zi, Jing Yuan, Binqiang Chen, and Zhengjia He. Wavelet transform based on inner product in fault diag- nosis of rotating machinery: A review. Mechanical systems and signal processing, 70:1-35, 2016.

[15] Tomasz Figlus. 1592. the application of a continuous wavelet transform for diagnosing damage to the timing chain tensioner in a motorcycle engine. Journal of Vibroengineering, 17(3), 2015.

[16] D Siano and D Dagostino. Knock detection in si engines by using the discrete wavelet transform of the engine block vibrational signals. Energy Procedia, 81:673-688, 2015.

[17] Daniela Siano, Maria Antonietta Panza, and Danilo D'Agostino. Knock detection based on mapo analysis, ar model and discrete wavelet transform applied to the in-cylinder pressure data: results and comparison. SAE International Journal of Engines, 8(1):1-13, 2015.

[18] Fengrong Bi, Lin Li, Jian Zhang, and Teng Ma. Source identification of gasoline engine noise based on continuous wavelet transform and eemd-robustica. Applied Acoustics, 100:34-42, 2015.

[19] Jian-Da Wu and Jun-Ming Kuo. An automotive generator fault diagnosis system using discrete wavelet transform and artificial neural network. Expert Systems with Applications, 36(6):97769783, 2009.

[20] Jian-Da Wu and Chiu-Hong Liu. An expert system for fault diagnosis in internal combustion engines using wavelet packet transform and neural network. Expert systems with applications, 36(3):4278-4286, 2009.

[21] Jian-Da Wu and Jien-Chen Chen. Continuous wavelet transform technique for fault signal diagnosis of internal combustion engines. NDT \& e International, 39(4):304-311, 2006.

[22] Humberto Guti'errez Pulido, Roman De la Vara Salazar, Porfirio Guti'errez Gonzalez, Carlos T'ellez Mart'inez, and Mar'́a del Carmen Temblador P'erez. Analisis y diseno de experimentos. McGraw-Hill New York, NY, USA:, 2012.

[23] Silvia Rocio Esparza Becerra. Analisis eficiente de camaras semianecoicas en alta frecuencia. 2012.

[24] Mechanical Vibration. Evaluation of machine vibration by measurements on non- rotating parts-part 6, 1995 